\title{
Estudo pHmétrico esofágico de oito horas em pacientes pediátricos com suspeita de refluxo gastroesofágico. ${ }^{1}$
}

\author{
Esophageal ph monitoring study of eight hours in pediatric patients with suspected \\ gastroesophageal reflux.
}

\author{
Ricardo Carneluti², José Luiz Martins ${ }^{3}$, Edson Khodor Cury ${ }^{4}$ \\ 1. Trabalho realizado no Curso de Pós-graduação da Disciplina de Cirurgia Pediátrica-Departmento de Cirurgia - Universidade \\ Federal de São Paulo (UNIFESP-EPM) - Brasil \\ 2. Mestre em Cirurgia Pediátrica pelo Curso de Pós-graduação da Disciplina de Cirurgia Pediátrica- Departmento de \\ Cirurgia - Universidade Federal de São Paulo (UNIFESP-EPM) - Brasil \\ 3. Professor Adjunto-Livre Docente Chefe da Disciplina de Pediátrica-Departmento de Cirurgia - Universidade Federal de \\ São Paulo (UNIFESP-EPM) - Brasil \\ 4. Professor Adjunto Doutor da Disciplina de Cirurgia Pediátrica- Departmento de Cirurgia - Universidade Federal de São \\ Paulo (UNIFESP-EPM) - Brasil
}

\section{RESUMO}

Objetivo: Estudar a pHmetria em oito horas, analisando variáveis como a idade, sintomatologia e esôfago-estômagoduodenografia, além dos seguintes parâmetros pHmétricos: número de episódios de refluxo, número de episódios maiores de cinco minutos, tempo de refluxo nas oito horas e episódio de maior duração, para o diagnóstico do refluxo gastroesofágico. Método: Estudo prospectivo realizado com a medição contínua do pH esofágico em oito horas em 35 pacientes, de quatro meses a 11 anos de idade. Foi utilizado aparelho de pHmetria DPS-100 Diagnosis pHmeter System com programa de acordo com a metodologia sugerida por Vandenplas e comparados com todas as variáveis e parâmetros do exame. Resultados: Obtivemos diferença estatisticamente significante em todos os parâmetros pHmétricos analisados, com o índice de refluxo sendo o mais efetivo para identificar o refluxo patológico, independente da idade. Todas as variáveis qualitativas, idade, sintomatologia e esôfago-estômago-duodenografia, não mostraram diferenças estatisticamente significantes quando relacionados aos resultados da pHmetria. Conclusão: Concluímos que a pHmetria esofágica de oito horas tem seus parâmetros pHmétricos válidos para o diagnóstico do refluxo gastroesofágico.

Descritores: Refluxo Gastroesofágico.

\begin{abstract}
Purpose: To study the pH monitoring over eight hours, analyzing variables such symptomatology and esophagogastroduodenoscopy, as well as the following $\mathrm{pH}$ monitoring parameters: number of reflux episodes, number of episodes longer than five minutes, length of reflux time during the eight hours, and the episode of longest duration, for the diagnosis of gastroesophageal reflux. Methods: A prospective study. Continuous measurement of esophageal pH was performed over eight hours on 35 patients whose ages ranged from four months to 11 years. The DPS-100 Diagnosis pH meter System was utilized, programmed in accordance with the methodology suggested by Vandenplas. The $\mathrm{pH}$ monitoring results were divided into positive and negative and compared with all the variables and parameters of the examination. Results: We obtained statistically significant differences in all the $\mathrm{pH}$ monitoring parameters analyzed, with the reflux index being the most effective for identifying the pathological reflux, independent of age. None of the qualitative variables of age, symptomatology and esophagogastroduodenoscopy showed statistically significant differences when compared to the $\mathrm{pH}$ monitoring results. Conclusion: The esophageal $\mathrm{pH}$ monitoring of eight hours has its $\mathrm{pH}$ monitoring parameters validated for the diagnosis of gastroesophageal reflux.
\end{abstract}

Key words: Gastroesophageal reflux.

\section{Introdução}

A pHmetria esofágica contínua seria o melhor método para diagnosticar o refluxo gastroesofágico, obtendo-se a melhor informação sobre o refluxo e sua associação com sintomas extra-intestinais ${ }^{1}$. O exame seria o único que pode detectar e quantificar o refluxo além do período pós-prandial² .
Foi confirmado que a pHmetria seria bem tolerada pelos pacientes e não apresentava complicações, devendo ser a primeira escolha como exame para o diagnóstico de refluxo gastroesofágico ${ }^{3}$.

A técnica da pHmetria é simples e os resultados padronizados em termos de análise. Porém, a realização em crianças de variadas idades tem um inconveniente sério, já 
que o paciente necessita ficar internado por 24 horas para o exame, alterando seu cotidiano, o que poderia levar a um resultado não confiável da pHmetria.

Também a falta de leitos hospitalares limita em muito a realização do exame. Em nosso meio, há poucos pHmetros portáteis para uso nos pacientes em casa, e, além disso, devido ao baixo nível cultural da maioria dos pais dos pacientes, há a necessidade de supervisão qualificada constante.

Por esses motivos, foi aventada hipótese de abreviarse o exame para menos de 24 horas.

Foi demonstrado que a pHmetria abreviada de oito horas apresentava resultados correlatos aos de 24 horas, recomendando este período mais curto para uso diagnóstico ${ }^{4,5}$.

Em crianças de seis a dez semanas de vida, devido à menor influência de variação de fatores como alimentação, posição e estado de vigília, a pHmetria de 12 horas pode ser realizada com resultados confiáveis, comparando-se com o estudo de 24 horas $^{6}$.

Em um estudo de 6 horas, foi evidenciado que haveria possibilidade de razoável confiabilidade dos resultados em crianças acima de 1 ano de idade ${ }^{7}$.

O refluxo gastroesofágico sintomático poderia ser confirmado em estudos pHmétricos abreviados de oito horas embora, em crianças com sintomas respiratórios, seria mais aconselhável a realização do exame de 24 horas $^{8}$.

Baseado nesses fatos, postulamos a realização de um estudo pHmétrico de oito horas para tentar resolver os problemas advindos da longa duração do exame de 24 horas. Relacionamos a idade, sintomatologia e esôfago-estômagoduodenografia como variáveis mais importantes na comparação com os resultados pHmétricos.

Objetivou-se estudar crianças com suspeita de refluxo gastroesofágico com pHmetria abreviada de oito horas pela metodologia de Vandenplas, uma das mais utilizadas em crianças, relacionando-a com a idade, a sintomatologia e a esôfago-estômago-duodenografia.

\section{Métodos}

Foram examinados 35 pacientes na Disciplina de Cirurgia Pediátrica da Universidade Federal de São Paulo (UNIFESP-EPM), com idade que variou de quatro meses a 11 anos, sendo 22 do sexo masculino e 13 do sexo feminino.

Todos os pacientes tinham suspeita de refluxo gastroesofágico, pelo quadro clínico ou complicações decorrentes, sendo seguidos ou encaminhados ao ambulatório da especialidade no período de 22/04/97 a 2/ 12/98.

Sete crianças submeteram-se a correção cirúrgica prévia de refluxo gastroesofágico, porém continuavam com sintomatologia ou complicações relativas ao refluxo. Desses, três tinham correção cirúrgica de atresia de esôfago realizada anteriormente.

Todos os pacientes sintomáticos fizeram tratamento clínico com medicação, dieta e/ou medidas posturais.

Os pacientes apresentavam-se sem jejum prévio, pela manhã, sendo aferidos dados antropométricos para cálculo do comprimento do fio do sensor a ser colocado nos pacientes. Era locado o fio neutro em eletrodo na coxa do paciente e iniciada calibração do sensor, de cerca de 1,5 mm de diâmetro, colocando-se em soluções de pH dois, quatro e sete, simultaneamente, a ponta do sensor e um dedo da criança. Se uma diferença de mais de 0,2 unidades $\mathrm{pH}$ ocorria, havia troca do sensor.

A seguir, o sensor lubrificado com lidocaína gel a 2\% era introduzido por via nasal até seu local no esôfago. Este local era determinado segundo a equação $C(\mathrm{~cm})=5+0,252$ $\mathrm{x} A(\mathrm{~cm})$, onde $\mathrm{C}$ é o comprimento do fio do sensor a partir da narina e A é a altura do paciente ${ }^{9}$.

Com esta fórmula, o sensor ficaria localizado de três a cinco $\mathrm{cm}$ acima do esfíncter esofágico inferior, a aproximadamente $87 \%$ da distância da narina à junção esofagogástrica. Quando havia dúvidas era realizada radiografia para o correto posisionamento do sensor.

Foi utilizado o pHmetro DPS 100 com um canal de registro e programa próprio, versão 2.0, instalado em um microcomputador 486 DX2, além de eletrodos de vidro (MI 506 Microelectrodes Inc., E.U.A), com vida útil média de 15 exames. O programa, além de capturar as aferições de $\mathrm{pH}$ esofágico, solicitava, a cada 30 minutos, informações sobre o decúbito e o estado de vigília do paciente, e, a qualquer tempo, permitia anotações ao longo do traçado tais como mudanças de decúbito, vigília, ingesta, tosse, choro e outras alterações do paciente.

Todos os dados referentes ao paciente e ao exame pHmétrico eram impressos em forma de relatório contendo anotações, gráficos e conclusões.

Foi adotada a metodologia de Vandenplas no programa, que liberava a dieta e o decúbito, posteriormente analisando os dados obtidos sob esse padrão. Após oito horas era finalizado o exame, sendo retirados o sensor e o fio neutro do paciente, que era liberado para casa. A Figura 1 mostra uma criança realizando o teste de phmetria esofágica.

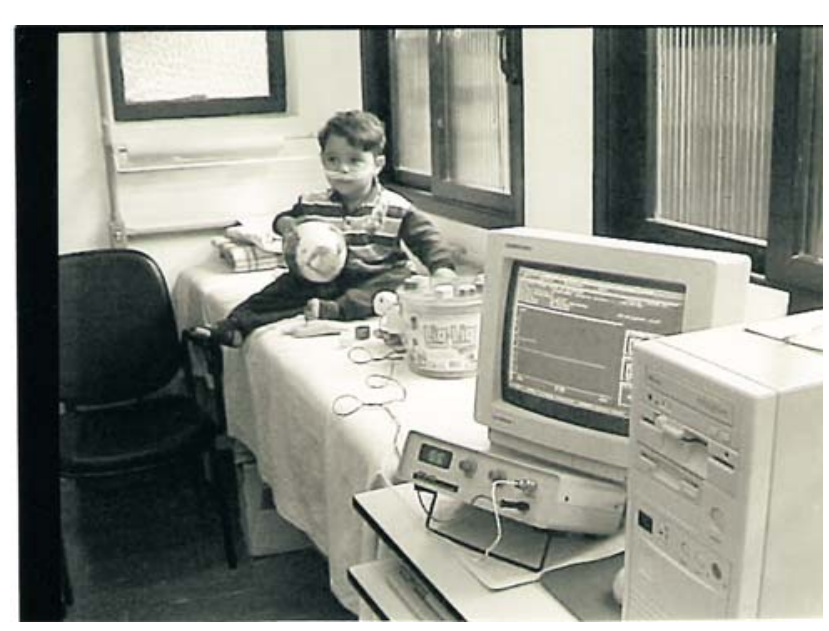

FIGURA 1- Criança submetida ao teste de phmetria esofágica.

A análise pela metodologia de Vandenplas consistia na utilização de quatro parâmetros, quais sejam, número de episódios de pH abaixo de quatro, duração do episódio mais longo com $\mathrm{pH}$ abaixo de quatro, porcentagem de tempo com $\mathrm{pH}$ abaixo de quatro (índice de refluxo) e número de episódios de $\mathrm{pH}$ abaixo de quatro por mais de cinco minutos. 
O programa do computador calculava todos esses parâmetros, relacionando os dados do paciente e apresentava a conclusão do exame, se positivo (em quais parâmetros) ou negativo. Os índices pHmétricos de normalidade adotados pelo aparelho dependiam da idade e dados antropométricos dos pacientes. Em média, o IR (índice de refluxo) para crianças acima de um ano era de 8,18, e, em crianças abaixo de um ano, ia de 2,5 até seis; o $\mathrm{N}$ total (número total de episódios de refluxo) variava de 10 a 11, com desvio-padrão de +/- 7; o N>5' (número de episódios de refluxo maiores de cinco minutos), cerca de 1 , com desviopadrão de $+/$ - 1; e o > T (duração do episódio de refluxo mais longo), 5,25', com desvio-padrão de +/- 4,7.

Os pacientes foram divididos em dois grupos, com pHmetria positiva e com pHmetria negativa, que foram comparados com as variáveis idade, sintomatologia e resultado da esôfago-estômago-duodenografia. Também foram analisados os quatro parâmetros utilizados para o diagnóstico pHmétrico já citados.

O estudo estatístico utilizado para variáveis qualitativas (idade, sintomatologia e resultado da esôfago-estômagoduodenografia), em tabelas de freqüência absoluta, e quantitativas (parâmetros pHmétricos), em tabelas com média, desvio padrão e intervalo, foram, respectivamente, o teste de qui-quadrado ou o exato de Fischer, e a prova paramétrica de Mann-Whitney. Adotou-se um nível de significância de $5 \%$, com $\mathrm{p}<0,05$.

Este estudo foi analisado e aprovado pela Comissão de Ética da UNIFESP/EPM (protocolo de pesquisa 583/98).

\section{Resultados}

Dos 35 pacientes submetidos ao exame, 15 tiveram pHmetria com resultado positivo para refluxo gastroesofágico e 20 com resultado negativo; dos positivos, quatro tinham menos de um ano e 11 mais de um ano de idade enquanto que dos negativos, quatro eram menores de um ano e 16 maiores de um ano.

Em relação às variáveis qualitativas confrontadas ao resultado da pHmetria, positivo ou negativo, obtivemos resultados sem significância estatística em todas comparações, conforme tabelas $\mathrm{pH}$ x idade, $\mathrm{pH}$ x sintomatologia e pH x EED (esôfago-estômagoduodenografia).

Já quanto às variáveis quantitativas dos parâmetros pHmétricos utilizados, tivemos resultados significantes estatisticamente na comparação $\mathrm{pH}$ x parâmetros pHmétricos. Houveram diferenças estatisticamente significantes comparando os resultados do exame, se positivo ou negativo, em relação a todos os parâmetros estudados: índice de refluxo (porcentagem de tempo com pH abaixo de 4), total de episódios de refluxo, total de episódios de refluxo com mais de cinco minutos e duração do episódio de refluxo mais longo (Tabela 1).

Analisamos também os parâmetros pHmétricos em relação à idade dos pacientes mostrando resultados estatisticamente significantes. Nas crianças abaixo de um ano de idade, apenas o parâmetro total de episódios de refluxo com mais de cinco minutos não teve significância estatística; todos os outros parâmetros foram estatisticamente significantes. Nos pacientes acima de um ano de idade, exceto o parâmetro total de episódios de refluxo, todos os parâmetros tiveram significância estatística (Tabelas 2 e 3).

TABELA 1 - Resultado da pHmetria x parâmetros pHmétricos

\begin{tabular}{|c|c|c|c|c|c|c|c|c|}
\hline \multirow[b]{2}{*}{ Parâmetros } & \multicolumn{3}{|c|}{ pHmetria positiva } & \multicolumn{3}{|c|}{ pHmetria negativa } & \multirow[b]{2}{*}{$\mathbf{U}$} & \multirow[b]{2}{*}{$\mathbf{p}$} \\
\hline & média & $\mathbf{\phi}$ & intervalo & média & $\mathbf{\phi}$ & intervalo & & \\
\hline IR total & 15,85 & 19,99 & $(1,93-73,85)$ & 1,54 & 1,67 & $(0-5,68)$ & 220 & $<0,000$ \\
\hline $\mathrm{N}$ total & 9,8 & 12,08 & $(1-44)$ & 3,2 & 3,35 & $(0-13)$ & 69 & 0,02 \\
\hline$N>5^{\prime}$ & 2,67 & 3,62 & $(0-15)$ & 0,1 & 0,31 & $(0-1)$ & 165 & $<0,000$ \\
\hline$>\mathrm{T}$ & 30,67 & 27,14 & $(5-90)$ & 3,35 & 2,92 & $(0-11)$ & 354 & $<\mathbf{0 , 0 0 0}$ \\
\hline
\end{tabular}

$\mathrm{U}=$ Mann-Whitney

TABELA 2 - Resultado da pHmetria x parâmetros de pacientes $<1$ ano

\begin{tabular}{|c|c|c|c|c|c|c|c|c|}
\hline \multirow[b]{2}{*}{ Parâmetros } & \multicolumn{3}{|c|}{ pHmetria positiva } & \multicolumn{3}{|c|}{ pHmetria negativa } & \multirow[b]{2}{*}{$\mathbf{U}$} & \multirow[b]{2}{*}{$\mathbf{p}$} \\
\hline & média & dp & intervalo & média & dp & intervalo & & \\
\hline IR total & 17,67 & 17,51 & $(5,53-43,36)$ & 0,32 & 0,7 & $(0-3)$ & 10 & $<0,05$ \\
\hline $\mathrm{N}$ total & 6,75 & 3,59 & $(4-12)$ & 0,75 & 1,5 & $(0-3)$ & 10 & $<0,05$ \\
\hline $\mathrm{N}>5^{\prime}$ & 2,25 & 1,71 & $(0-4)$ & 0 & 0 & $(0-0)$ & 12 & $>0,05$ \\
\hline$>\mathrm{T}$ & 39,25 & 36,2 & $(5-90)$ & 1,25 & 2,5 & $(0-5)$ & 11 & $<0,05$ \\
\hline
\end{tabular}


TABELA 3 - Resultados da pHmetria x parâmetros de pacientes $\geq 1$ ano

\begin{tabular}{|c|c|c|c|c|c|c|c|c|}
\hline \multirow[b]{2}{*}{ Parâmetros } & \multicolumn{3}{|c|}{ pHmetria positiva } & \multicolumn{3}{|c|}{ pHmetria negativa } & \multirow[b]{2}{*}{$\mathbf{U}$} & \multirow[b]{2}{*}{ p } \\
\hline & média & dp & intervalo & média & dp & intervalo & & \\
\hline IR total & 15,15 & 21,6 & $(1,93-73,85)$ & 1,85 & 1,7 & $(0-5,68)$ & 224 & $<0,000$ \\
\hline $\mathrm{N}$ total & 10,42 & 13,4 & $(1-44)$ & 3,81 & 3,4 & $(0-13)$ & 191 & 0,069 \\
\hline $\mathrm{N}>5^{\prime}$ & 2,82 & 4,17 & $(1-15)$ & 0,13 & 0,3 & $(0-1)$ & 235 & $<0,000$ \\
\hline$>\mathrm{T}$ & 27,55 & 24,5 & $(6-83)$ & 3,88 & 2,9 & $(0-11)$ & 235 & $<0,000$ \\
\hline
\end{tabular}

LEGENDA

IR = Índice de Refluxo - porcentagem de tempo com $\mathrm{pH}<4$

$\mathrm{N}$ total $=$ total de episódios com $\mathrm{pH}<4$

$\mathrm{N}>5^{\prime}$ = total de episódios com $\mathrm{pH}<4$ por mais de 5 minutos

$>\mathrm{T}=$ duração do episódio mais longo com $\mathrm{pH}<4$

$\mathrm{dp}=$ desvio padrão

$\mathrm{p}$ = nível de significância

\section{Discussão}

Embora bastante heterogênea em relação à idade, intervenções anteriores, além de sintomatologia bastante diversificada, esta casuística apresentava um ponto crucial em comum: todos os pacientes tinham suspeita diagnóstica de refluxo gastroesofágico. Os pacientes assintomáticos por ocasião do exame de pHmetria haviam tido complicações recentes importantes, como crises de broncoespasmo ou broncopneumonia.

Provavelmente devido à heterogeneidade e tamanho da casuística, observou-se que todas as variáveis qualitativas não foram significantes estatisticamente.

Todos os procedimentos foram realizados como preconizou Vandemplas, exceto o tempo do exame, de oito horas.

Analisando peso, altura e superfície corporal dos pacientes, foi demonstrado que a altura seria o parâmetro mais fidedigno para se chegar ao comprimento do esôfago. Através de uma fórmula matemática na qual utilizou-se a altura foi calculada a localização do eletrodo esofágico ${ }^{9}$.

O pH limite indicativo de refluxo foi quatro, já consagrado por vários autores ${ }^{10,11}$, havendo indícios que há início de sintomatologia de dor retroesternal quando se tinha níveis de $\mathrm{pH}$ abaixo de quatro.

Em crianças foram utilizados os seguintes parâmetros na pHmetria: a) a porcentagem de tempo com $\mathrm{pH}$ menor que quatro (índice de refluxo); b) a duração do maior episódio com pH menor que quatro; c) o número de episódios de $\mathrm{pH}$ menor que quatro e d) o número de episódios com mais de cinco minutos com pH menor que quatro. A duração do episódio de refluxo combinado com a acidez seriam os maiores determinantes da esofagite péptica ${ }^{2}$.

Apesar do programa de computador nos permitir a opção de várias metodologias, preferimos a de Vandenplas para a realização e cálculo dos resultados da pHmetria, pois mantinha, na medida do possível, o paciente dentro de sua rotina normal, com dieta, posicionamento e estado de vigília livres.

Comparando-se a pHmetria esofágica de oito e 24 horas durante o mesmo exame e no mesmo paciente, autores concluíram que o exame feito por 8 horas era representativo do exame de 24 horas, podendo ser utilizado para diagnosticar o refluxo gastroesofágico ${ }^{4,5}$.

Optou-se então por adotar a pHmetria abreviada de oito horas no grupo de esôfago da Disciplina de Cirurgia Pediátrica da Universidade Federal de São Paulo. Assim, poder-se-ia minimizar os custos do exame bem como realizálo ambulatorialmente, com maior conforto para o paciente.

Os pacientes foram distribuídos em grupo positivo e negativo, dependendo do resultado obtido na pHmetria ${ }^{12}$. Estes resultados foram analisados em comparação com diversas variáveis qualitativas como a sintomatologia, e quantitativas, em relação aos parâmetros do próprio exame.

Notou-se que, nos nossos resultados, dos diferentes parâmetros utilizados, analisando também a idade como variável, o índice de refluxo e o episódio mais longo, foram os mais efetivos para demonstrar o refluxo gastroesofágico, seguidos dos parâmetros número de episódios com $\mathrm{pH}<4$ maiores de cinco minutos e do número total de episódios com $\mathrm{pH}<4$.

Embora não se pode correlacionar diretamente a sintomatologia com os resultados da pHmetria abreviada de oito horas utilizada com a metodologia de Vandenplas, os parâmetros pHmétricos por nós utilizados foram suficientemente confiáveis para podermos diagnosticar o refluxo gastroesofágico independente das variáveis.

\section{Conclusão}

A pHmetria abreviada de oito horas pela metodologia de Vandenplas tem seus parâmetros pHmétricos válidos para o diagnóstico de refluxo gastroesofágico. 


\section{Referências}

1 - Hart JJ: Pediatric gastroesophageal reflux. Am Fam Physician. 1996; 54:2463-9.

2 - Vandenplas Y, Loeb H. The interpretation of oesophageal pH monitoring data. Eur J Pediatr. 1990; 149:598-602.

3 - Dalt L, Mazzoleni S, Montini G, Donzelli F, Zacchello F. Diagnostic accuracy of $\mathrm{pH}$ monitoring in gastrooesophageal reflux. Arch Dis Child. 1989; 64:1421-6.

4 - Choniere L, Miller L, Ilves R. Comparison of 8 hour studies with 24 hour studies. In: T.R. DeMeester, D.B. Skinner, eds. Esophageal disorders: pathophysiology and therapy. New York: Raven Press, 1985; pp.583-8

5 - Silva JMB, Silva PEMR, Brunelli MMC. PHmetría esofágica abreviada. Correlación de valores del índice de reflujo (IR) en exámenes de 8 y 24 hs. Rev Cir Infant. 1996; 6:208-12.

6 - Vandenplas Y, Casteels A, Naert M, Derde MP, Blecker U. Abbreviated oesophageal $\mathrm{pH}$ monitoring in infants. Eur J Pediatr. 1994; 153:80-3.

7 - Barabino A, Costantini M, Ciccone MO, Pesce F, Parodi
B, Gatti R. Reliability of short-term esophageal $\mathrm{pH}$ monitoring versus 24-hour study. J Pediatr Gastroenterol Nutr. 1995; 21:87-90.

8 - Jolley SG, Tunell WP, Carson, Smith EI, Grunow J. The accuracy of abbreviated esophageal $\mathrm{pH}$ monitoring in children. J Pediatr Surg. 1984; 19:848-54.

9- Strobel CT, Byrne WJ, Ament ME, Euler AR. Correlation of esophageal lengths in children with height: Application to the Tuttle test without prior esophageal manometry. J Pediatr. 1979; 94:81-4.

10 - Johnsson LR, DeMeester TR. Twenty-four-hour pH monitoring of the distal esophagus. A quantitative measure of gastro-oesophageal reflux. Am J Gastroenterol. 1974; 62:323-32.

11 - Johnsson F, Joelson B, Isberg, PE. Ambulatory 24-hour intraesophageal $\mathrm{pH}$ monitoring in the diagnosis of gastroesophageal reflux disease. Gut. 1987; 28:1145-50.

12 - Durante AP, Costa HGC, Iasi M, Neto PN. PHmetría esofágica prolongada (24 hs.) en la evaluación del reflujo gastroesofágico en niños. Rev Cir Infant. 1996; 6:200-7.
Correspondência:

Ricardo Carneluti

R. dos Otonis, 880 ap. 83 - São Paulo - SP - Brasil

CEP 04025-002

phone/fax: 55-011-55759276E-mail:carneluti@brfree.com.br
Conflito de interesse: nenhum Fonte de financiamento: nenhuma

Recebimento: $13 / 10 / 2004$

Revisão: 25/11/2004

Aprovação: 28/12/2004

\section{Como citar este artigo:}

Carneluti R, Martins JL, Cury EK. Estudo pHmétrico esofágico de oito horas em pacientes pediátricos com suspeita de refluxo gastroesofágico. Acta Cir Bras. [serial online] 2005 Mar-Abr;20(2). Disponível em URL: http://www.scielo.br/acb 